GLASNIK MATEMATIČKI

Vol. 41(61)(2006), $77-88$

\title{
A NOTE ON REDUCTION OF DIMENSION FOR LINEAR ELLIPTIC EQUATIONS
}

\author{
Ibrahim Aganović, Josip Tambača And Zvonimir Tutek \\ University of Zagreb, Croatia
}

\begin{abstract}
In this paper we consider the linear elliptic equation of the second order in domains in $\mathbb{R}^{n}$ thin in $n-k$ directions, $0<k<n$. We apply the Ansatz of the formal expansion method (with respect to the small parameter (thickness)) which implies the scaling of the loads in the equation that allows the reduction of the problem from dimension $n$ to dimension $k$. Appropriate convergence result together with correctors is derived.
\end{abstract}

\section{INTRODUCTION}

Reduction of dimension for the Laplace equation was considered in [1] and [8] and, for general linear elliptic equation of the second order (assuming the reduction from dimension $n$ to $n-1$ ) in [7]. Very general nonlinear monotone equation was analyzed in [6]. We consider here the case of linear elliptic equation of the second order, but assuming the reduction from dimension $n$ to dimension $k, 0<k<n$.

The success in obtaining a lower-dimensional model without restriction (compatibility condition) on source terms depends essentially on scaling them (and coefficients) with respect to the small parameter (a thickness of the domain). In the mentioned works (as in other earlier papers on asymptotic analysis, see $[2,5,10]$ ) an a priori scaling was taken in order to guarantee the existence of a low-dimensional model and convergence results. Using an idea of the paper [9] we show here that an appropriate scaling is a consequence of the Ansatz of formal expansion method (see [3, pp. 89-95, 269]). We also prove a convergence theorem and find the first correctors.

2000 Mathematics Subject Classification. 34E05, 35J25.

Key words and phrases. Reduction dimension, elliptic equation, formal expansion method, Ansatz. 


\section{Statement of the Problem and the main Result}

Let $n, k \in \mathbb{N}, 0<k<n, \mathbb{R}^{n}=\mathbb{R}_{x}^{k} \times \mathbb{R}_{y}^{n-k}$. Let $\omega \subset \mathbb{R}_{x}^{k}$ and $\sigma \subset \mathbb{R}_{y}^{n-k}$ be bounded regular domain and

$$
\Omega=\omega \times \sigma, \quad \gamma=\partial \sigma, \quad \Gamma=\omega \times \gamma, \quad \Gamma_{0}=\partial \omega \times \sigma .
$$

For sufficiently small $\varepsilon>0$ we define

$$
\sigma^{\varepsilon}=\varepsilon \sigma, \quad \gamma^{\varepsilon}=\partial \sigma^{\varepsilon}, \quad \Omega^{\varepsilon}=\omega \times \sigma^{\varepsilon}, \quad \Gamma^{\varepsilon}=\omega \times \gamma^{\varepsilon}, \quad \Gamma_{0}^{\varepsilon}=\partial \omega \times \sigma^{\varepsilon} .
$$

Evidently the transformation

$$
\left(x^{\varepsilon}, y^{\varepsilon}\right)=(x, \varepsilon y)
$$

is a bijection as a function from $\Omega \rightarrow \Omega^{\varepsilon}, \Gamma \rightarrow \Gamma^{\varepsilon}$ and $\Gamma_{0} \rightarrow \Gamma_{0}^{\varepsilon}$.

Let $\mathcal{A}^{\varepsilon} \in L^{\infty}\left(\Omega^{\varepsilon} ; \operatorname{Sym}(n)\right)$ and $a_{0}^{\varepsilon} \in L^{\infty}\left(\Omega^{\varepsilon}\right)$. We assume that for each $\varepsilon>0$ the matrix $\mathcal{A}^{\varepsilon}$ is positive definite (uniformly on $\Omega^{\varepsilon}$ ) and $a_{0}^{\varepsilon} \geq 0$ on $\Omega^{\varepsilon}$. Let

$$
V^{\varepsilon}=\left\{v \in H^{1}\left(\Omega^{\varepsilon}\right): v=0 \text { on } \Gamma_{0}^{\varepsilon}\right\} .
$$

For given $f^{\varepsilon} \in L^{2}\left(\Omega^{\varepsilon}\right)$ and $g^{\varepsilon} \in L^{2}\left(\Gamma^{\varepsilon}\right)$ we shall consider a boundary value problem: find $u^{\varepsilon} \in V^{\varepsilon}$ such that for each $v^{\varepsilon} \in V^{\varepsilon}$ it holds

$$
\int_{\Omega^{\varepsilon}}\left(\mathcal{A}^{\varepsilon} \nabla u^{\varepsilon} \cdot \nabla v^{\varepsilon}+a_{0}^{\varepsilon} u^{\varepsilon} v^{\varepsilon}\right) d x^{\varepsilon} d y^{\varepsilon}=\int_{\Omega^{\varepsilon}} f^{\varepsilon} v^{\varepsilon} d x^{\varepsilon} d y^{\varepsilon}+\int_{\Gamma^{\varepsilon}} g^{\varepsilon} v^{\varepsilon} d x^{\varepsilon} d \gamma^{\varepsilon} .
$$

This problem has unique solution by the Lax-Milgram lemma.

The matrix $\mathcal{A}^{\varepsilon}$ can be written in the form

$$
\mathcal{A}^{\varepsilon}=\left(\begin{array}{cc}
a^{\varepsilon} & \left(b^{\varepsilon}\right)^{T} \\
b^{\varepsilon} & c^{\varepsilon}
\end{array}\right),
$$

where $a^{\varepsilon}, b^{\varepsilon}$ and $c^{\varepsilon}$ are respectively $k \times k,(n-k) \times k$ and $(n-k) \times(n-$ $k$ ) matrices. Note that the matrices $a^{\varepsilon}$ and $c^{\varepsilon}$ are positive definite as a consequence of positivity of $\mathcal{A}^{\varepsilon}$. The problem (2.1) then takes the form: find $u^{\varepsilon} \in V^{\varepsilon}$ such that

$$
\begin{aligned}
& \int_{\Omega^{\varepsilon}}\left(a^{\varepsilon} \nabla_{x^{\varepsilon}} u^{\varepsilon} \cdot \nabla_{x^{\varepsilon}} v^{\varepsilon}+\left(b^{\varepsilon}\right)^{T} \nabla_{y^{\varepsilon}} u^{\varepsilon} \cdot \nabla_{x^{\varepsilon}} v^{\varepsilon}+b^{\varepsilon} \nabla_{x^{\varepsilon}} u^{\varepsilon} \cdot \nabla_{y^{\varepsilon}} v^{\varepsilon}\right. \\
& \left.\quad+c^{\varepsilon} \nabla_{y^{\varepsilon}} u^{\varepsilon} \cdot \nabla_{y^{\varepsilon}} v^{\varepsilon}+a_{0}^{\varepsilon} u^{\varepsilon} v^{\varepsilon}\right) d x^{\varepsilon} d y^{\varepsilon} \\
& =\int_{\Omega^{\varepsilon}} f^{\varepsilon} v^{\varepsilon} d x^{\varepsilon} d y^{\varepsilon}+\int_{\Gamma^{\varepsilon}} g^{\varepsilon} v^{\varepsilon} d x^{\varepsilon} d \gamma^{\varepsilon}, \quad v^{\varepsilon} \in V^{\varepsilon} .
\end{aligned}
$$

For a function $v^{\varepsilon}$ defined on $\Omega^{\varepsilon}$ we define the function $v(\varepsilon)$ on $\Omega$ by the composition

$$
v(\varepsilon)(x, y)=v^{\varepsilon}(x, \varepsilon y)
$$

Then, if $v^{\varepsilon}$ belongs respectively to the space $L^{\infty}\left(\Omega^{\varepsilon}\right), L^{2}\left(\Omega^{\varepsilon}\right), L^{2}\left(\Gamma^{\varepsilon}\right)$ and $V^{\varepsilon}$, the function $v(\varepsilon)$ belongs to the space $L^{\infty}(\Omega), L^{2}(\Omega), L^{2}(\Gamma)$ and

$$
V=\left\{v \in H^{1}(\Omega): v=0 \text { on } \Gamma_{0}\right\} \text {. }
$$


The following fact is an immediate consequence of (2.3).

LEMMA 2.1. The problem (2.2) is equivalent to the problem: find $u(\varepsilon) \in V$ such that

$$
\begin{aligned}
& \int_{\Omega}\left(\varepsilon a(\varepsilon) \nabla_{x} u(\varepsilon) \cdot \nabla_{x} v+b(\varepsilon)^{T} \nabla_{y} u(\varepsilon) \cdot \nabla_{x} v+b(\varepsilon) \nabla_{x} u(\varepsilon) \cdot \nabla_{y} v\right. \\
& \left.\quad+\varepsilon^{-1} c(\varepsilon) \nabla_{y} u(\varepsilon) \cdot \nabla_{y} v+\varepsilon a_{0}(\varepsilon) u(\varepsilon) v\right) d x d y \\
& =\varepsilon \int_{\Omega} f(\varepsilon) v d x d y+\int_{\Gamma} g(\varepsilon) v d x d \gamma, \quad v \in V .
\end{aligned}
$$

Assuming that the coefficients on the left hand side are of the same order in $\varepsilon$, without loss of generality (see Ciarlet [4, p. 58]) one can take

$$
\begin{gathered}
a(\varepsilon)=a, \quad b(\varepsilon)=b, \quad c(\varepsilon)=c, \quad a_{0}(\varepsilon)=a_{0}, \\
f(\varepsilon)=\varepsilon^{p} f^{p}, \quad g(\varepsilon)=\varepsilon^{p+1} g^{p+1},
\end{gathered}
$$

where $p \in \mathbb{Z}, a, b, c, a_{0}, f^{p}$ and $g^{p+1}$ do not depend on $\varepsilon$ (here superscripts on $f$ and $g$ are not exponents, but denote the order of the force with respect to $\varepsilon)$. The equation (2.4) takes the form

$$
\begin{aligned}
& \int_{\Omega}\left(\varepsilon a \nabla_{x} u(\varepsilon) \cdot \nabla_{x} v+b^{T} \nabla_{y} u(\varepsilon) \cdot \nabla_{x} v+b \nabla_{x} u(\varepsilon) \cdot \nabla_{y} v+\varepsilon^{-1} c \nabla_{y} u(\varepsilon) \cdot \nabla_{y} v\right. \\
& \left.(2.6)+\varepsilon a_{0} u(\varepsilon) v\right) d x d y=\varepsilon^{p+1} \int_{\Omega} f^{p} v d x d y+\varepsilon^{p+1} \int_{\Gamma} g^{p+1} v d x d \gamma, \quad v \in V .
\end{aligned}
$$

The form of the problem (2.6) suggests the following Ansatz of formal expansion method:

A.1 There exists a number $l \in \mathbb{Z}$ such that for each $\left(f^{p}, g^{p+1}\right) \in L^{2}(\Omega) \times$ $L^{2}(\Gamma)$ there exist functions $u^{l}, u^{l+1}, \ldots \in H^{1}(\Omega)$, not depending on $\varepsilon$, such that for a solution $u(\varepsilon)$ of the problem $(2.4),(2.5)$ there holds

$$
u(\varepsilon)=\varepsilon^{l} u^{l}+\varepsilon^{l+1} u^{l+1}+\cdots,
$$

where the leading term $u^{l}$ is nontrivial for at least one pair $\left(f^{p}, g^{p+1}\right)$.

A.2 The successive terms $u^{m}, m=l, l+1, \ldots$ in (2.7) satisfy the equations obtained (after inserting (2.7) into (2.4)) by the cancellations of the coefficients of $\varepsilon^{m}, m \in \mathbb{Z}$.

A.3 The leading term $u^{l}$ belongs to the space $V$.

Because of the linearity of the problem (2.4) one can take $l=0$, i.e.

$$
u(\varepsilon)=u^{0}+\varepsilon^{1} u^{1}+\cdots .
$$

An inspection of coefficients of different powers of $\varepsilon$ that appear in (2.4) (under assumption (2.5)) shows that the pair $\left(f^{p}, g^{p+1}\right)$ is trivial if $p<-2$. Our purpose is to find the smallest number $p$ for which the pair $\left(f^{p}, g^{p+1}\right)$ is not necessary trivial and to identify the corresponding leading term $u^{0}$. We shall prove the following results. 
THEOREM 2.2. If for each pair $(f, g)$ there exist terms $u^{0}, u^{1}, u^{2}$ of the expansion (2.8), then $p=0$, i.e.

$$
f(\varepsilon)=f^{0}, \quad g(\varepsilon)=\varepsilon g^{1} .
$$

The leading term $u^{0}$ has the form

$$
u^{0}(x, y)=U^{0}(x)
$$

$U^{0} \in H_{0}^{1}(\omega)$ is the unique solution to the problem:

$$
\int_{\omega}\left(A \nabla_{x} U^{0} \cdot \nabla_{x} \eta+a_{0} U^{0} \eta\right) d x=\int_{\omega} F \eta d x, \quad \eta \in H_{0}^{1}(\omega)
$$

where

$$
\begin{aligned}
A & =\int_{\sigma}\left(a+\left(\nabla_{y} \boldsymbol{w} b\right)^{T}\right) d y \\
A_{0} & =\int_{\sigma} a_{0} d y \\
F & =\int_{\sigma} f^{0} d y+\int_{\gamma} g^{1} d \gamma ;
\end{aligned}
$$

here $\boldsymbol{w} \in L^{\infty}\left(\omega ; H^{1}(\sigma)^{k}\right)$ is the unique solution of the problem:

$$
\int_{\sigma}\left(\nabla_{y} \boldsymbol{w} c^{T}+b^{T}\right) \nabla_{y} \theta d y=0, \quad \theta \in H^{1}(\sigma), \quad \int_{\sigma} \boldsymbol{w} d y=0 .
$$

REMARK 2.3. The auxiliary problem (2.11) can be formulated for components of $\boldsymbol{w}$ : find $w_{i} \in L^{\infty}\left(\omega ; H^{1}(\sigma)\right)$ such that

$$
\int_{\sigma}\left(c \nabla_{y} w_{i}+b_{i}\right) \cdot \nabla_{y} \theta d y=0, \quad \theta \in H^{1}(\sigma), \quad \int_{\sigma} w_{i} d y=0,
$$

where $b_{i}$ is $i$ th column of the matrix $b$. As $c$ is positive definite (uniformly on $\Omega)$ this problem has unique solution.

Remark 2.4. Positive definiteness of $\mathcal{A}=\mathcal{A}^{\varepsilon}$ implies that for all $v_{x} \in \mathbb{R}^{k}$ one has (denote $\left.v=\left(v_{x}, \nabla_{y} w b^{T} v_{x}\right)\right)$ :

$$
\begin{aligned}
m_{\mathcal{A}}\|v\|^{2} & \leq \mathcal{A} v \cdot v \\
& =a v_{x} \cdot v_{x}+b^{T} \nabla_{y} \boldsymbol{w}^{T} v_{x} \cdot v_{x}+b v_{x} \cdot \nabla_{y} \boldsymbol{w}^{T} v_{x}+c \nabla_{y} \boldsymbol{w}^{T} v_{x} \cdot \nabla_{y} \boldsymbol{w}^{T} v_{x} \\
& =a v_{x} \cdot v_{x}+2 b^{T} \nabla_{y} \boldsymbol{w}^{T} v_{x} \cdot v_{x}+\nabla_{y} \boldsymbol{w} c \nabla_{y} \boldsymbol{w}^{T} v_{x} \cdot v_{x} \\
& =\left(a+2 b^{T} \nabla_{y} \boldsymbol{w}^{T}+\nabla_{y} \boldsymbol{w} c \nabla_{y} \boldsymbol{w}^{T}\right) v_{x} \cdot v_{x} .
\end{aligned}
$$

Taking the integral over $\sigma$ one gets

$$
m_{\mathcal{A}}|\sigma|\left\|v_{x}\right\|^{2} \leq \int_{\sigma}\left(a+2 b^{T} \nabla_{y} \boldsymbol{w}^{T}+\nabla_{y} \boldsymbol{w} c \nabla_{y} \boldsymbol{w}^{T}\right) d y v_{x} \cdot v_{x} .
$$

Inserting $w_{j}$ for the test function in (2.12) we obtain

$$
\int_{\sigma}\left(c \nabla_{y} w_{i} \cdot \nabla_{y} w_{j}+b_{i} \cdot \nabla_{y} w_{j}\right) d y=0
$$


This equation can be written as

$$
\int_{\sigma}\left(\nabla_{y} \boldsymbol{w} c \nabla_{y} \boldsymbol{w}^{T}+b^{T} \nabla_{y} \boldsymbol{w}^{T}\right) d y=0 .
$$

Using this in (2.13) we obtain that $A$ is positive definite uniformly on $\omega$ and therefore the problem (2.10) has unique solution.

Remark 2.5. One can easily see that in the case $k=n-1(n>1)$ there holds

$$
A_{i j}=\int_{\sigma}\left(a_{i j}-\frac{b_{i} b_{j}}{c}\right) d y, \quad i, j=1,2, \ldots, n-1 .
$$

TheOREM 2.6. Let

$$
u^{0, \varepsilon}\left(x^{\varepsilon}, y^{\varepsilon}\right)=U^{0}\left(x^{\varepsilon}\right), \quad\left(x^{\varepsilon}, y^{\varepsilon}\right) \in \Omega^{\varepsilon} .
$$

Then

$$
\frac{1}{\left|\Omega^{\varepsilon}\right|^{1 / 2}}\left\|u^{\varepsilon}-u^{0, \varepsilon}\right\|_{L^{2}\left(\Omega^{\varepsilon}\right)} \rightarrow 0, \quad \varepsilon \rightarrow 0 .
$$

Note that $\left|\Omega^{\varepsilon}\right|=\varepsilon^{n-k}|\Omega|$.

REMARK 2.7. In order to get the appropriate convergence of the approximation in $H^{1}\left(\Omega^{\varepsilon}\right)$ (actually the convergence of the gradient in $\left.L^{2}\left(\Omega^{\varepsilon}\right)^{n}\right)$ one needs to involve the corrector into the approximation:

$$
u^{1, \varepsilon}\left(x^{\varepsilon}, y^{\varepsilon}\right)=U^{0}\left(x^{\varepsilon}\right)+\varepsilon \boldsymbol{w}\left(\frac{y^{\varepsilon}}{\varepsilon}\right) \cdot \nabla_{x^{\varepsilon}} U^{0}\left(x^{\varepsilon}\right) .
$$

For this approximation we need higher smoothness of the coefficients in the equations to be able to apply the regularity result. Moreover, the boundary condition is no longer satisfied for the approximation.

REMARK 2.8. The result (2.9) shows that the ratio between (some appropriate measure) of body (surface) source term and coefficients must behave like $\varepsilon^{0}\left(\varepsilon^{1}\right)$.

As we assumed, without loss of generality, that the expansions for the coefficients $\mathcal{A}(\varepsilon)$ and the solutions $u(\varepsilon)$ start with the power 0 , we can now go back and restate the previous conclusion. Denote

$$
\begin{aligned}
\mathcal{A}(\varepsilon) & =\varepsilon^{r} \mathcal{A}, \\
u(\varepsilon) & =\varepsilon^{l} u^{l}+\varepsilon^{l+1} u^{l+1}+\cdots, \\
f(\varepsilon) & =\varepsilon^{p} f^{p}, \quad g(\varepsilon)=\varepsilon^{p+1} g^{p+1} .
\end{aligned}
$$

Then the result (2.9) shows that

$$
p-r-l=0 .
$$

Then, for instance, we can draw the following interpretation: if the loads $(p)$ are too strong $(p-r<0)$ for the material $(r)$, then the solution is singular $(l<0)$. 


\section{Proof of Theorem 2.2}

The equations for successive terms of expansion (2.8) will be found by the use of assumptions A.2 and A.3. We shall divide the proof into three steps.

STEP 1 . As we noticed before, $p \geq-2$. Let $p=-2$, i.e.

$$
f(\varepsilon)=\varepsilon^{-2} f^{-2}, \quad g(\varepsilon)=\varepsilon^{-1} g^{-1} .
$$

By cancellation of the coefficient of $\varepsilon^{-1}$ in (2.6) we obtain

$$
\int_{\Omega} c \nabla_{y} u^{0} \cdot \nabla_{y} v d x d y=\int_{\Omega} f^{-2} v d x d y+\int_{\Gamma} g^{-1} v d x d \gamma .
$$

Setting $v(x, y)=\eta(x), \eta \in H_{0}^{1}(\omega)$ we have

$$
\int_{\Omega} f^{-2} \eta d x d y+\int_{\Gamma} g^{-1} \eta d x d \gamma=0
$$

or equivalently

$$
\int_{\sigma} f^{-2} d y+\int_{\gamma} g^{-1} d \gamma=0
$$

This is in contradiction with the assumption A.1 that $u^{0}$ exists for an arbitrary $\left(f^{-2}, g^{-1}\right)$; therefore

$$
p \geq-1
$$

Setting now in (2.4) $v(x, y)=\eta(x) \theta(y), \eta(x) \in H_{0}^{1}(\omega), \theta \in H^{1}(\sigma)$ and taking into account (3.2), we obtain

$$
\int_{\sigma} c \nabla_{y} u^{0} \cdot \nabla_{y} \theta d y=0
$$

because of positive definiteness of $c$ we conclude that

$$
u^{0}(x, y)=U^{0}(x)
$$

where (because of A.3)

$$
U^{0} \in H_{0}^{1}(\omega)
$$

STEP 2. Because of (3.2) we take $p=-1$, i.e.

$$
f(\varepsilon)=\varepsilon^{-1} f^{-1}, \quad g(\varepsilon)=g^{0} .
$$

By the cancellation of the coefficient of $\varepsilon^{0}$ in (2.6) we obtain for all $v \in V$

$$
\int_{\Omega}\left(b \nabla_{x} u^{0} \cdot \nabla_{y} v+c \nabla_{y} u^{1} \cdot \nabla_{y} v\right) d x d y=\int_{\Omega} f^{-1} v d x d y+\int_{\Gamma} g^{0} v d x d \gamma
$$

Setting $v(x, y)=\eta(x), \eta \in H_{0}^{1}(\omega)$ and taking into account (3.3) we have

$$
\int_{\sigma} f^{-1} d y+\int_{\gamma} g^{0} d \gamma=0
$$

and therefore

$$
p \geq 0 .
$$


Setting now in (3.4) $v(x, y)=\eta(x) \theta(y), \eta(x) \in H_{0}^{1}(\omega), \theta \in H^{1}(\sigma)$ and taking into account (3.3) and (3.5) we obtain

$$
\int_{\sigma}\left(b \nabla_{x} U^{0} \cdot \nabla_{y} \theta+c \nabla_{y} u^{1} \cdot \nabla_{y} \theta\right) d y=0
$$

From (3.6) we conclude that there exists $U^{1} \in H^{1}(\omega)$ such that

$$
u^{1}=U^{1}+\boldsymbol{w} \cdot \nabla_{x} U^{0},
$$

where $\boldsymbol{w}$ is a solution of the problem (2.11).

STEP 3. Because of (3.5) we take

$$
f(\varepsilon)=f^{0}, \quad g(\varepsilon)=\varepsilon g^{1} .
$$

By the cancellation of the coefficient of $\varepsilon^{1}$ in (2.6) we obtain

$$
\begin{gathered}
\int_{\Omega}\left(a \nabla_{x} u^{0} \cdot \nabla_{x} v+b^{T} \nabla_{y} u^{1} \cdot \nabla_{x} v+b \nabla_{x} u^{1} \cdot \nabla_{y} v+c \nabla_{y} u^{2} \cdot \nabla_{y} v\right. \\
\left.+a_{0} u^{0} v\right) d x d y=\int_{\Omega} f^{0} v d x d y+\int_{\Gamma} g^{1} v d x d \gamma, \quad v \in V .
\end{gathered}
$$

Setting $v(x, y)=\eta(x), \eta \in H_{0}^{1}(\omega)$ and taking into account (3.3) and (3.7) we have

$$
\begin{aligned}
\int_{\Omega} & \left(a \nabla_{x} U^{0} \cdot \nabla_{x} \eta+b^{T} \nabla_{y} \boldsymbol{w}^{T} \nabla_{x} U^{0} \cdot \nabla_{x} \eta+a_{0} U^{0} \eta\right) d x d y \\
= & \int_{\Omega} f^{0} \eta d x d y+\int_{\Gamma} g^{1} \eta d x d \gamma
\end{aligned}
$$

and hence (2.10).

\section{Proof of Theorem 2.6}

For $f(\varepsilon)=f^{0}$ and $g(\varepsilon)=\varepsilon g^{1}$ we are able to prove a priori estimates, uniform with respect to $\varepsilon$, for the solution $u(\varepsilon)$ of (2.4). These estimates imply weak convergence of $u(\varepsilon)$ toward a function which is uniquely determined as a solution of (2.10).

Uniform positive definiteness of $\mathcal{A}$ implies that there is $m_{\mathcal{A}}>0$ such that

$$
\int_{\Omega} \mathcal{A}\left(\begin{array}{c}
\nabla_{x} v \\
\frac{1}{\varepsilon} \nabla_{y} v
\end{array}\right) \cdot\left(\begin{array}{c}
\nabla_{x} v \\
\frac{1}{\varepsilon} \nabla_{y} v
\end{array}\right) d x d y \geq m_{\mathcal{A}}\left(\left\|\nabla_{x} v\right\|_{L^{2}(\Omega)}^{2}+\frac{1}{\varepsilon^{2}}\left\|\nabla_{y} v\right\|_{L^{2}(\Omega)}^{2}\right),
$$

for all $v \in V$. Application of this estimate to (2.4), divided by $\varepsilon$, using (2.9), implies

$$
\begin{aligned}
m_{\mathcal{A}} & \left(\left\|\nabla_{x} u(\varepsilon)\right\|_{L^{2}(\Omega)}^{2}+\frac{1}{\varepsilon^{2}}\left\|\nabla_{y} u(\varepsilon)\right\|_{L^{2}(\Omega)}^{2}\right) \\
& \leq \int_{\Omega} f^{0} u(\varepsilon) d x d y+\int_{\Gamma} g^{1} u(\varepsilon) d x d \gamma \\
& \leq\left\|f^{0}\right\|_{L^{2}(\Omega)}\|u(\varepsilon)\|_{L^{2}(\Omega)}+\left\|g^{1}\right\|_{L^{2}(\Gamma)}\|u(\varepsilon)\|_{L^{2}(\Gamma)} .
\end{aligned}
$$


Application of the Poincaré inequality and continuity of the trace implies

$$
m_{\mathcal{A}}\left(\left\|\nabla_{x} u(\varepsilon)\right\|_{L^{2}(\Omega)}^{2}+\frac{1}{\varepsilon^{2}}\left\|\nabla_{y} u(\varepsilon)\right\|_{L^{2}(\Omega)}^{2}\right)^{1 / 2} \leq\left\|f^{0}\right\|_{L^{2}(\Omega)}+\left\|g^{1}\right\|_{L^{2}(\Gamma)},
$$

which implies the a priori estimates uniform with respect to $\varepsilon$

$$
\exists C>0, \quad\|u(\varepsilon)\|_{L^{2}(\Omega)} \leq C,\left\|\nabla_{x} u(\varepsilon)\right\|_{L^{2}(\Omega)^{k}} \leq C, \frac{1}{\varepsilon}\left\|\nabla_{y} u(\varepsilon)\right\|_{L^{2}(\Omega)^{n-k}} \leq C .
$$

These estimates imply that there exist a subsequence, still denoted by $u(\varepsilon)$, and functions $u \in L^{2}(\Omega)$ and $\gamma \in L^{2}(\Omega)^{n-k}$ such that

$$
\begin{aligned}
& u(\varepsilon) \rightarrow u \quad \text { weakly in } L^{2}(\Omega), \\
& \nabla_{x} u(\varepsilon) \rightarrow \nabla_{x} u \quad \text { weakly in } L^{2}(\Omega)^{k}, \\
& \frac{1}{\varepsilon} \nabla_{y} u(\varepsilon) \rightarrow \gamma \quad \text { weakly in } L^{2}(\Omega)^{n-k} .
\end{aligned}
$$

The last convergence implies

$$
\nabla_{y} u(\varepsilon) \rightarrow 0 \quad \text { strongly in } L^{2}(\Omega)^{n-k}
$$

and by uniqueness of the limit function $\nabla_{y} u=0$. Hence there exists a function $U \in L^{2}(\omega)$ such that

$$
u(x, y)=U(x) .
$$

Moreover convergence (4.2) imply that

$$
u(\varepsilon) \rightarrow u \quad \text { weakly in } V .
$$

Therefore $u \in V$ and consequently $U \in H_{0}^{1}(\omega)$.

We can take more information out of (4.2). Take $\varphi \in H_{0}^{1}(\Omega)^{n-k}$ such that $\operatorname{div}_{y} \varphi=0$. Then from (4.2) it follows

$$
0=-\frac{1}{\varepsilon} \int_{\Omega} u(\varepsilon) \operatorname{div}_{y} \varphi=\int_{\Omega} \frac{1}{\varepsilon} \nabla_{y} u(\varepsilon) \cdot \varphi \rightarrow \int_{\Omega} \gamma \cdot \varphi .
$$

Therefore there is $\Phi \in L^{2}\left(\omega ; H^{1}(\sigma)\right)$ such that

$$
\gamma=\nabla_{y} \Phi
$$

Now we identify $U$ more precisely.

STEP 1. Take the limit $\varepsilon \rightarrow 0$ in (2.4). The limit equation is

$$
\int_{\Omega} b \nabla_{x} U \cdot \nabla_{y} v+c \gamma \cdot \nabla_{y} v=0, \quad v \in V .
$$

Let us define

$$
\boldsymbol{\gamma}^{H}=\boldsymbol{\gamma}-\nabla_{y}\left(\boldsymbol{w} \cdot \nabla_{x} U\right),
$$

where $\boldsymbol{w} \in H^{1}(\omega)^{k}$ is defined in (2.11). Insertion of this $\boldsymbol{\gamma}$ into (4.5) implies the equation for $\gamma^{H}$ :

$$
\int_{\Omega} c \boldsymbol{\gamma}^{H} \cdot \nabla_{y} v=0, \quad v \in V
$$


As $\gamma^{H}$ is of the form (4.4) as well, this equation and positive definiteness of $c$ imply $\gamma^{H}=0$. Therefore

$$
\gamma=\nabla_{y}\left(\boldsymbol{w} \cdot \nabla_{x} U\right)
$$

STEP 2. Setting $v(x, y)=\eta(x), \eta \in H_{0}^{1}(\omega)$ in (2.4), dividing it by $\varepsilon$, and taking $\varepsilon \rightarrow 0$ imply

$$
\int_{\Omega}\left(a \nabla_{x} U \cdot \nabla_{x} \eta+b^{T} \gamma \cdot \nabla_{x} \eta+a_{0} U \eta\right) d x d y=\int_{\omega} F \eta d x .
$$

Inserting the obtained form of $\gamma$ we obtain (2.10). By uniqueness of the solution of (2.10) one has $U=U^{0}$. Moreover, the whole family $u(\varepsilon)$ converges to $U^{0}$.

Let us define the approximation of the starting problem (2.1), on a $\varepsilon$ dependent domain:

$$
u^{0, \varepsilon}\left(x^{\varepsilon}, y^{\varepsilon}\right)=U^{0}\left(x^{\varepsilon}\right)
$$

Then (4.4) implies

$$
\frac{1}{\left|\Omega^{\varepsilon}\right|^{1 / 2}}\left\|u^{\varepsilon}-u^{0, \varepsilon}\right\|_{L^{2}\left(\Omega^{\varepsilon}\right)}=\frac{1}{|\Omega|^{1 / 2}}\left\|u(\varepsilon)-U^{0}\right\|_{L^{2}(\Omega)} \rightarrow 0 .
$$

This concludes the proof of Theorem 2.6.

4.1. The strong convergence in $H^{1}\left(\Omega^{\varepsilon}\right)$. In order to get the strong convergence in $H^{1}\left(\Omega^{\varepsilon}\right)$ we need the strong convergence of the gradient. Inspection of (4.2) forces the definition of the approximation

$$
u^{1}(\varepsilon)(x, y)=U^{0}(x)+\varepsilon \boldsymbol{w}(y) \cdot \nabla_{x} U^{0}(x) .
$$

Here we assume additional smoothness of the coefficients in (2.10) so additional regularity of the solution, $U^{0} \in H^{2}(\omega)$, is obtained. One has

$$
\begin{aligned}
\int_{\Omega} \mathcal{A} \nabla^{\varepsilon}\left(u(\varepsilon)-u^{1}(\varepsilon)\right) \cdot \nabla^{\varepsilon}\left(u(\varepsilon)-u^{1}(\varepsilon)\right)+a_{0}\left(u(\varepsilon)-u^{1}(\varepsilon)\right)^{2} \\
=\int_{\Omega} f^{0} u(\varepsilon)+\int_{\Gamma} g^{1} u(\varepsilon) \\
\left.\quad-2 \int_{\Omega} \mathcal{A}\left(\begin{array}{c}
\nabla_{x} u(\varepsilon) \\
\frac{1}{\varepsilon} \nabla_{y} u(\varepsilon)
\end{array}\right) \cdot\left(\begin{array}{c}
\nabla_{x} U^{0}+\varepsilon \nabla_{x}\left(\boldsymbol{w} \cdot \nabla_{x} U^{0}\right) \\
\nabla_{y} \boldsymbol{w}^{T} \nabla_{x} U^{0}
\end{array}\right)+a_{0} u(\varepsilon) u^{1}(\varepsilon)\right) \\
\quad+\int_{\Omega} \mathcal{A}\left(\begin{array}{c}
\nabla_{x} U^{0}+\varepsilon \nabla_{x}\left(\boldsymbol{w} \cdot \nabla_{x} U^{0}\right) \\
\nabla_{y} \boldsymbol{w}^{T} \nabla_{x} U^{0}
\end{array}\right) \cdot\left(\begin{array}{c}
\nabla_{x} U^{0}+\varepsilon \nabla_{x}\left(\boldsymbol{w} \cdot \nabla_{x} U^{0}\right) \\
\nabla_{y} \boldsymbol{w}^{T} \nabla_{x} U^{0}
\end{array}\right) \\
\quad+a_{0}\left(U^{0}+\varepsilon \boldsymbol{w} \cdot \nabla_{x} U^{0}\right)^{2} \\
\rightarrow \int_{\omega} F U^{0}-2 \int_{\Omega} \mathcal{A}\left(\begin{array}{c}
\nabla_{x} U^{0} \\
\nabla_{y} \boldsymbol{w}^{T} \nabla_{x} U^{0}
\end{array}\right) \cdot\left(\begin{array}{c}
\nabla_{x} U^{0} \\
\nabla_{y} \boldsymbol{w}^{T} \nabla_{x} U^{0}
\end{array}\right)+a_{0}\left(U^{0}\right)^{2} \\
\quad+\int_{\Omega} \mathcal{A}\left(\begin{array}{c}
\nabla_{x} U^{0} \\
\nabla_{y} \boldsymbol{w}^{T} \nabla_{x} U^{0}
\end{array}\right) \cdot\left(\begin{array}{c}
\nabla_{y} \boldsymbol{w}^{T} \nabla_{x} U^{0}
\end{array}\right)+a_{0}\left(U^{0}\right)^{2}=: L .
\end{aligned}
$$


For the limit $L$ it follows

$$
\begin{aligned}
L & =\int_{\omega} F U^{0}-\int_{\Omega} \mathcal{A}\left(\begin{array}{c}
\nabla_{x} U^{0} \\
\nabla_{y} \boldsymbol{w}^{T} \nabla_{x} U^{0}
\end{array}\right) \cdot\left(\begin{array}{c}
\nabla_{x} U^{0} \\
\nabla_{y} \boldsymbol{w}^{T} \nabla_{x} U^{0}
\end{array}\right)+a_{0}\left(U^{0}\right)^{2} \\
& =-\int_{\Omega}\left(b \nabla_{x} U^{0}+c \nabla_{y} \boldsymbol{w}^{T} \nabla_{x} U^{0}\right) \cdot \nabla_{y}\left(\boldsymbol{w}^{T} \nabla_{x} U^{0}\right) \\
& =-\int_{\Omega}\left(b+c \nabla_{y} \boldsymbol{w}^{T}\right) \nabla_{x} U^{0} \cdot \nabla_{y}\left(\boldsymbol{w}^{T} \nabla_{x} U^{0}\right) \\
& =-\int_{\Omega} \nabla_{x} U^{0} \cdot\left(b^{T}+\nabla_{y} \boldsymbol{w} c^{T}\right) \nabla_{y}\left(\boldsymbol{w}^{T} \nabla_{x} U^{0}\right) \\
& =-\int_{\omega} \nabla_{x} U^{0} \cdot \int_{\sigma}\left(b^{T}+\nabla_{y} \boldsymbol{w} c^{T}\right) \nabla_{y}\left(\boldsymbol{w}^{T} \nabla_{x} U^{0}\right)=0 .
\end{aligned}
$$

We define

$$
u^{1, \varepsilon}\left(x^{\varepsilon}, y^{\varepsilon}\right)=u^{0, \varepsilon}\left(x^{\varepsilon}, y^{\varepsilon}\right)+\varepsilon \boldsymbol{w}\left(\frac{y^{\varepsilon}}{\varepsilon}\right) \cdot \nabla_{x^{\varepsilon}} u^{0, \varepsilon}\left(x^{\varepsilon}, y^{\varepsilon}\right) .
$$

As $\mathcal{A}$ is uniformly positive definite one has

$$
\begin{aligned}
& \frac{1}{\left|\Omega^{\varepsilon}\right|}\left\|\nabla u^{\varepsilon}-\nabla u^{1, \varepsilon}\right\|_{L^{2}\left(\Omega^{\varepsilon}\right)^{n}}^{2} \\
& \quad=\left\|\nabla_{x} u(\varepsilon)-\nabla_{x} u^{1}(\varepsilon)\right\|_{L^{2}(\Omega)^{k}}^{2}+\frac{1}{\varepsilon^{2}}\left\|\nabla_{y} u(\varepsilon)-\nabla_{y} u^{1}(\varepsilon)\right\|_{L^{2}(\Omega)^{n-k}}^{2} \rightarrow 0,
\end{aligned}
$$

as $\varepsilon \rightarrow 0$.

REMARK 4.1. If $b^{\varepsilon}=0$, then $\boldsymbol{w}=0$ and

$$
\frac{1}{\left|\Omega^{\varepsilon}\right|}\left\|\nabla u^{\varepsilon}-\nabla u^{0, \varepsilon}\right\|_{L^{2}\left(\Omega^{\varepsilon}\right)^{n}}^{2} \rightarrow 0
$$

so no corrector is necessary.

\section{THE " OVERLOOKED" MODELS}

If $p<0$, then the pair $\left(f^{p}, g^{p+1}\right)$ must satisfy the compatibility condition

$$
\int_{\sigma} f^{p} d y+\int_{\gamma} g^{p+1} d \gamma=0
$$

If we neglect the assumption A.1 of the Ansatz, the pair $\left(f^{p}, g^{p+1}\right)$ is not necessary trivial. The analysis shows that if $p<-1$, there appear new complicated compatibility conditions without a clear interpretation. Therefore we shall assume $p=-1$. Let

$$
\int_{\sigma} f^{-1} d y+\int_{\gamma} g^{0} d \gamma=0
$$


Then (3.3) and (3.4) hold true. Let $U^{1} \in L^{2}\left(\omega ; H^{1}(\sigma) \cap L_{0}^{2}(\sigma)\right)$ be the unique solution to the problem

$$
\int_{\sigma} c \nabla_{y} U^{1} \cdot \nabla_{y} \theta d y=\int_{\sigma} f^{-1} \theta d y+\int_{\gamma} g^{0} \theta d \gamma
$$

From (3.4) it follows

$$
u^{1}=z^{1}+U^{1}+\boldsymbol{w} \cdot \nabla_{x} U^{0}
$$

where

$$
z^{1} \in H^{1}(\omega)
$$

By the cancellation of the coefficient of $\varepsilon^{1}$ in (2.6) and taking into account (3.3) and (5.2) we conclude that $U^{0}$ is a unique solution to the problem

$$
\int_{\omega}\left(\left(A \nabla_{x} U^{0}+B\right) \cdot \nabla_{x} \eta+a_{0} U^{0} \eta\right) d x=0, \quad \eta \in H_{0}^{1}(\omega)
$$

where

$$
B=\int_{\sigma} b^{T} \nabla_{y} U^{1} d y
$$

\section{REFERENCES}

[1] I. Aganović, Z. Tutek, On the lower-dimensional approximations of the mixed problem for Laplace equation, Glasnik Mat. 20 (1985), 355-361.

[2] A. Bermudez, J. M. Viaño, A justification of thermoelastic equations for variablesection beams by asymptotic methods, RAIRO Anal. Numér. 18 (1984), 347-376.

[3] P. G. Ciarlet, Mathematical elasticity. Vol. II. Theory of plates. North-Holland Publishing Co., Amsterdam, 1997.

[4] P. G. Ciarlet, Plates and junctions in elastic multistructures. An asymptotic analysis, Masson, Paris, 1990.

[5] P. G. Ciarlet, P. Destuynder, A justification of the two dimensional linear plate model, J. Mécanique 18 (1979) 315-344.

[6] P. Courilleau and J. Mossino, Compensated compactness for nonlinear homogenization and reduction of dimension. Calc. Var. Partial Differential Equations 20 (2004), 65-91.

[7] B. Gustafsson and J. Mossino, Non-periodic explicit homogenization and reduction of dimension: the linear case. IMA J. Appl. Math. 68 (2003), 269-298.

[8] H. Le Dret, Problèmes variationnels dans les multi-domains, Masson, Paris, 1991.

[9] B. Miara, Justification of the asymptotic analysis of elastic plates. I. The linear case, Asymptotic Anal. 9 (1994), 47-60.

[10] Z. Tutek, I. Aganović, A justification of the one-dimensional linear model of elastic beam, Math. Methods Appl. Sci. 8 (1986) 502-515. 


\author{
I. Aganović \\ Department of Mathematics \\ University of Zagreb \\ Bijenička 30, 10000 Zagreb \\ Croatia \\ E-mail: aga@math.hr \\ J. Tambača \\ Department of Mathematics \\ University of Zagreb \\ Bijenička 30, 10000 Zagreb \\ Croatia \\ E-mail: tambaca@math.hr \\ Z. Tutek \\ Department of Mathematics \\ University of Zagreb \\ Bijenička 30, 10000 Zagreb \\ Croatia \\ E-mail: tutek@math.hr \\ Received: 14.7.2005. \\ Revised: 30.10.2005.
}

